\title{
Genomic and transcriptomic comparison of Aspergillus oryzae strains: a case study in soy sauce koji fermentation
}

\author{
Yiyi Zhong ${ }^{1} \cdot \mathrm{Xi} \mathrm{Lu}^{1} \cdot$ Lei Xing $^{2} \cdot$ Shiu Woon Allen $\mathrm{Ho}^{3} \cdot$ Hoi Shan Kwan ${ }^{1,2}$
}

Received: 16 May 2018 / Accepted: 18 June 2018 / Published online: 5 July 2018

(c) The Author(s) 2018

\begin{abstract}
The filamentous fungus Aspergillus oryzae is used in soy sauce koji making due to its high productivity of hydrolytic enzymes. In this study, we compared the genomes and transcriptomes of an industrial strain RD2 and a strain with decreased fermentation performance TS2, aiming to explain their phenotypic differences at the molecular level. Under the regulation of conidiation and fermentation conditions, the enhanced hydrolytic enzyme production and flavor precursor formation in $\mathrm{RD} 2$ described a complete expression profile necessary to maintain desirable fermentation performance. By contrast, central carbon metabolism was up-regulated in TS2 for fast growth, suggesting a conflicting relationship between mycelium growth and fermentation performance. Accumulation of mutations also lowered the fermentation performance of TS2. Our study has deepened the understanding of the metabolism and related regulatory mechanisms in desirable koji fermentation. A list of potential molecular markers identified here could facilitate targeted strain maintenance and improvement for better koji fermentation.
\end{abstract}

Keywords Aspergillus oryzae $\cdot$ Soy sauce $\cdot$ Koji fermentation $\cdot$ Genome $\cdot$ Transcriptome

\section{Introduction}

The filamentous fungus Aspergillus oryzae, also known as the koji mold, has been widely used in the production of traditional fermented food such as soy sauce, soybean paste and rice wine in China, Japan and other Asian countries for centuries. To make soy sauce, A. oryzae is cultured on a mixture of steamed soybeans and wheat flour. After a 3-day solid-state fermentation called koji fermentation, the resulting koji is then mixed with a brine solution for further submerged fermentation $[11,13]$. Used as the starter

Electronic supplementary material The online version of this article (https://doi.org/10.1007/s10295-018-2059-8) contains supplementary material, which is available to authorized users.

Hoi Shan Kwan

hoishankwan@cuhk.edu.hk

1 School of Life Sciences, The Chinese University of Hong Kong, Shatin, NT, Hong Kong SAR, China

2 Food Research Centre, The Chinese University of Hong Kong, Shatin, NT, Hong Kong SAR, China

3 Lee Kum Kee International Holdings Limited, Taipo, NT, Hong Kong SAR, China of koji fermentation, A. oryzae has a prominent potential of secreting various hydrolytic enzymes to break down large molecules such as polysaccharides and proteins into smaller monosaccharides and amino acids [17, 26, 44]. These enzymes are fundamental and indispensable in the fermentation process, allowing prolonged degradation of biomass to energize subsequent fermentation and to supply metabolites that act as precursors for the characteristic flavor formation of soy sauce $[9,23]$.

Since the release of the first genome sequence of $A$. oryzae (RIB40 ATCC-42149) in 2005 [26], efforts have been made to understand the metabolism of this industrially important species. Comparative genomic studies have demonstrated that genes related to metabolism, especially hydrolytic processes, are overexpressed in A. oryzae compared to other aspergilli due to an expansion of these metabolic genes $[17,26]$. Transcriptomic studies using microarray and RNA-Seq have revealed a high expression of metabolic genes in solid-state fermentation, thereby confirming the immense contribution of $A$. oryzae in fermentation as a source of enzymes and metabolites [28, 44]. For soy sauce, various $A$. oryzae strains have been isolated and selected for effective fermentation on soybeans, including the Japanese strain RIB326 [43] and the Chinese strain 3.042, which is 
equivalent to 3.951 [51]. These strains are common in producing a large number of hydrolytic enzymes, especially proteases, to adapt to the protein-rich environment during soybean fermentation, with acid protease possibly playing the most vital role in protein hydrolysis under the acidic environment of soy sauce fermentation [22, 48, 50]. High protease production can directly affect the amino acid content, and thus amino acid nitrogen, which is used to determine the quality grade of soy sauce in China [25].

Until now, the molecular mechanisms of A. oryzae metabolism and related regulations during soy sauce fermentation remain only partially understood, hindering targeted strain improvement and effective strain maintenance against industrial strain degeneration. For instance, although multiple hydrolases have been identified in soy sauce koji $[22,50]$, the transcriptional regulation of hydrolase production during koji fermentation remains unclear, not to mention the hydrolases that cannot be detected in proteomic approaches. To uncover the metabolism that is necessary to maintain koji fermentation performance, here we presented a case study of the comparison between a productive strain RD2 and strain TS2 that produced unfavorable soy sauce. Through integrated genomic and transcriptomic analysis, we identified a list of molecular markers and revealed the transcriptional regulatory mechanisms that are related to desirable koji fermentation.

\section{Materials and methods}

\section{Strains and cultivation}

Strains used in this study were obtained from a Chinese soy sauce factory: A. oryzae RD2 used in koji fermentation could produce good-quality soy sauce, whereas TS2 was isolated after repeated use of RD2, which produced soy sauce with a decreased amino acid yield and an unfavored flavor. The published genome of A. oryzae RIB40 [26] was downloaded from the Aspergillus Genome Database (AspGD) (http://www.aspergillusgenome.org/) (Version September 2016) and used as a reference.

RD2 and TS2 were cultivated on potato dextrose agar (PDA) plates at $30{ }^{\circ} \mathrm{C}$ for 3-4 days. Spores were collected and spread in autoclaved water to make an aqueous conidia suspension (ACS) $\left(10^{8}\right.$ spores $\left./ \mathrm{ml}\right)$, which was stored at $4{ }^{\circ} \mathrm{C}$ until further use.

To produce koji, soaked soybeans were steamed, mixed with wheat flour, and inoculated with the prepared ACS. Koji fermentation was performed at $30{ }^{\circ} \mathrm{C}$ for about $72 \mathrm{~h}$ at $\geq 85 \%$ humidity. The process can be divided into three stages depending on the fermentation time and morphological changes: stage I mycelia expansion (ME), spans about $28 \mathrm{~h}$, after which the surface of soybeans is covered with the white mycelia of $A$. oryzae; stage II early sporulation (ES), spans about $48 \mathrm{~h}$, after which yellow-green spores appear on the white mycelia; and stage III mature sporulation (MS), spans about $72 \mathrm{~h}$, after which the white mycelia is almost completely covered by spores, meaning that the koji is mature for subsequent fermentation.

\section{Strain characterization and enzyme activities}

The mycelium growth diameter (in mm) of RD2 and TS2 on PDA plates was measured in triplicate in a daily basis. Growth rate was calculated as the mycelium growth diameter divided by the number of days $(\mathrm{mm} / \mathrm{d})$.

Protease and amylase activities at the ME, ES and MS stages of koji fermentation were determined in triplicate. To prepare crude enzyme solution, $5 \mathrm{~g}$ koji samples were mixed with $45 \mathrm{ml}$ distilled water, then stirred at $40{ }^{\circ} \mathrm{C}$ for an hour. The filtered supernatant was centrifuged at $5000 \mathrm{~g}$ for $20 \mathrm{~min}$. The protease activity was assayed by a modified method of Kum et al. [20], using 2\% casein dissolved in $\mathrm{pH}$ 7.2 phosphate buffer $\left(0.1 \mathrm{M} \mathrm{Na}_{2} \mathrm{HPO}_{4}\right.$ plus $\left.0.1 \mathrm{M} \mathrm{KH}_{2} \mathrm{PO}_{4}\right)$ as substrate. A standard tyrosine solution was used to make a calibration curve for quantitative analysis. One unit of protease activity was defined as the quantity of protease that induced a change of $1 \mu \mathrm{g}$ of tyrosine from $2 \%$ casein solution per minute. Amylase activity was assayed by a modified method of Fuwa [10], using 1\% starch solution dissolved in pH 5.2 buffer $\left(0.1 \mathrm{M}\right.$ citric acid plus $\left.0.2 \mathrm{M} \mathrm{Na}_{2} \mathrm{HPO}_{4}\right)$ as substrate and measurement at $580 \mathrm{~nm}$ absorbance. One unit of amylase activity was referred to the quantity of amylase that catalyzed the conversion of $1 \%$ soluble starch solution to $1 \mathrm{mg}$ equivalent of maltose per minute.

$\mathrm{pH}$ of koji at the three stages was also determined from a well-mixed slurry of $5 \mathrm{~g}$ solution and $45 \mathrm{ml}$ distilled water using a benchtop pH meter (Thermo Fisher Scientific).

\section{DNA extraction and ion torrent sequencing}

Spores and mycelia of $A$. oryzae freshly grown on PDA plates at $30{ }^{\circ} \mathrm{C}$ for $3-4$ days were collected and ground into fine powder with a mortar and pestle in liquid nitrogen. Genomic DNA was then extracted using the DNeasy Plant Mini Kit (Qiagen). Fragment DNA library construction was performed using the Ion Plus Fragment Library Kit (Thermo Fisher Scientific). About 100 ng of genomic DNA was enzymatically sheared by a Bioruptor (Diagenode) and ligated to Ion Torrent adapters. Fragments of $480 \mathrm{bp}$ were selected by $2 \%$ agarose gel electrophoresis using the E-Gel iBase Power System and an E-Gel Safe Imager (Invitrogen, Thermo Fisher Scientific). Template for sequencing was prepared using the Ion OneTouch 2 System with the Ion PI Template OT2 400 Kit v2 (Thermo Fisher Scientific). Sequencing was performed on an Ion Torrent Personal Genome Machine 
(PGM 318 chip) using the Ion PGM Sequencing 400 Kit (Thermo Fisher Scientific).

\section{Genome mapping and variant calling}

Quality of the raw DNA-Seq reads was first evaluated by FastQC (v0.11.4) [2]. Poor-quality reads (with a base quality $<20$ in more than half of the bases) were filtered using the "fastq_quality_filter" command in the FASTX-Toolkit (http://hannonlab.cshl.edu/fastx_toolkit/). ShRiMP2 was then used to map the RD2 and TS2 genomes against RIB40, with the "-V" parameter adjusted from a previous method [7].

Uniquely mapped reads were used to perform single nucleotide variant (SNV) calling including single nucleotide polymorphisms (SNPs) and indel with the SAMtools "mpileup" command [21]. Only SNP-containing sites with at least three reads covering the position with an average quality $\geq 20$ and indel-containing sites with at least five reads covering the position with an average quality $\geq 20$ were extracted for further analysis. SNPeff [5] was then used to annotate the SNPs and indels with an SNPeff database created from RIB40 and custom parameters for fungal genomes.

\section{RNA extraction and Illumina HiSeq sequencing}

Spores and mycelia were collected in duplicate from the three stages of koji fermentation and ground into powder with a mortar and pestle in liquid nitrogen. RNA was extracted using the RNeasy Micro Kit (Qiagen). RNA integrity was evaluated using gel electrophoresis and a NanoDrop Lite Spectrophotometer (Thermo Fisher Scientific). Transcriptomes were then sequenced on an Illumina HiSeq 2000 sequencing system at BGI Genomics.

\section{Transcriptome mapping and differential expression analysis}

The raw RNA-Seq reads were first aligned to the A. oryzae RIB40 genome using TopHat. Cufflinks and Cuffdiff were then used to quantitatively normalize the expression levels to the number of fragments per kilobase of transcript per million mapped reads (FPKM) and to identify differentially expressed genes (DEGs) [42]. Genes with a fold change $\geq 1$ and false discovery rate (FDR) $<0.05$ were considered as significantly differentially expressed. The pheatmap package was used to generate heatmaps [18]. Functional analysis based on EuKaryotic Orthologous Groups (KOGs) was performed by subjecting DEGs to the WebMGA server (http://weizhong-lab.ucsd.edu/metagenomic-analysis) [47] using the kog functional annotation with an $e$ value $<0.001$. Pathway graphs were obtained from KEGG (http://www. genome.jp/kegg/).
Validation of RNA-Seq data by real-time RT-PCR

RNA extracted was used in real-time RT-PCR to verify the RNA-Seq result. Genomic DNA-free cDNA was synthesized using iScript gDNA Clear cDNA Synthesis Kit (Bio-Rad). Real-time RT-PCR was performed on an ABI 7500 Fast Real-Time PCR System (applied biosystems) with iQ SYBR Green Supermix (Bio-Rad). Primers were designed in the exon regions of six selected genes (Table $\mathrm{S} 1$ ) and the expression of these genes was evaluated. The beta-tubulin gene (btuA) was used as a reference to normalize gene expression levels, and the relative transcriptional levels of the six genes were calculated (Fig. S1).

\section{Nucleotide sequence accession numbers}

DNA-Seq and RNA-Seq data generated in this study are available in the sequence read archive (SRA) of NCBI under accession numbers SRP132444 and SRP132645, respectively.

\section{Results and discussion}

\section{Strain growth and enzyme activities}

RD2 and TS2 exhibited different phenotypes on PDA plates. TS2 possessed a significantly higher mycelium growth rate $(13.9 \pm 1.2 \mathrm{~mm} / \mathrm{d})$ compared to $\mathrm{RD} 2(11.8 \pm 1.8 \mathrm{~mm} / \mathrm{d})$ (Fig. 1b), showing a growth advantage of TS2 over RD2 and TS2 began conidiation at $32 \mathrm{~h}$, which is $4 \mathrm{~h}$ ahead of RD2. However, RD2 displayed complete conidiation with yellowgreen spores, whereas conidiation in TS2 was decreased, especially at the edge of colonies, with asporogenous mycelium left (white hyphae) and sclerotia formed (dark spots) (Fig. 1a). Sclerotia formation has been reported in A. ory$z a e$ as a result of aberrant branching and intertwining of mycelia [15]. Normally, after gemination of spores, A. ory$z a e$ acquires nutrients for mycelium or vegetative growth to penetrate the substrate, aerial hyphae then develop into conidiophores to perform conidiation [40]. Therefore, the decreased conidiation observed in TS2 is probably because of the fact that less aerial hyphae could successfully develop into conidiophores. Also, TS2 showed a faster growth than RD2 in the ME, ES, MS stages of koji fermentation, as it accomplished each stage in a shorter time, but with decreased conidiation especially in the MS stage (Fig. 2a).

The pattern of $\mathrm{pH}$ change differed in koji fermented by the two strains (Fig. 2b). In RD2 koji, pH first decreased from 6.5 to 6.0 in the ME stage, then increased gradually back to 6.5; while in TS2 koji, $\mathrm{pH}$ first declined from 6.5 to 5.8 in the ES stage, and then rose to 6.8 . The $\mathrm{pH}$ change in RD2 koji is similar to that reported in previous study [22]. 
Fig. 1 Growth phenotypes of RD2 and TS2 on PDA plates. a RD2 and TS2 grown after $72 \mathrm{~h}$. The arrow indicates a sclerotium (dark spot) produced by TS2. b Mycelium growth diameter and growth rate of RD2 and TS2. Data are presented as mean \pm standard deviation. Significant difference was detected between RD2 and TS2 in all days $(P<0.01$, Student's $t$ test $)$ a

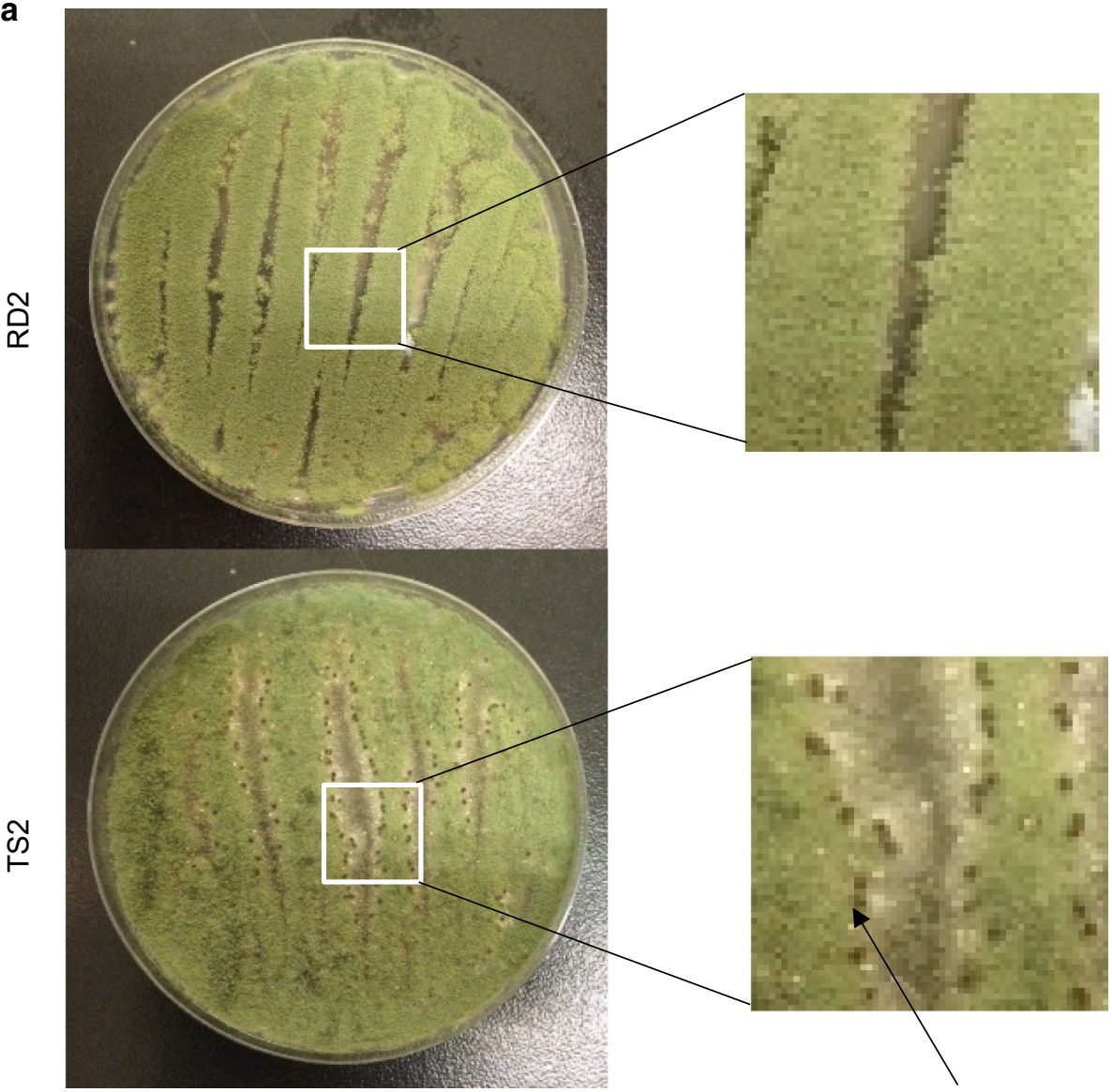

Sclerotium

b

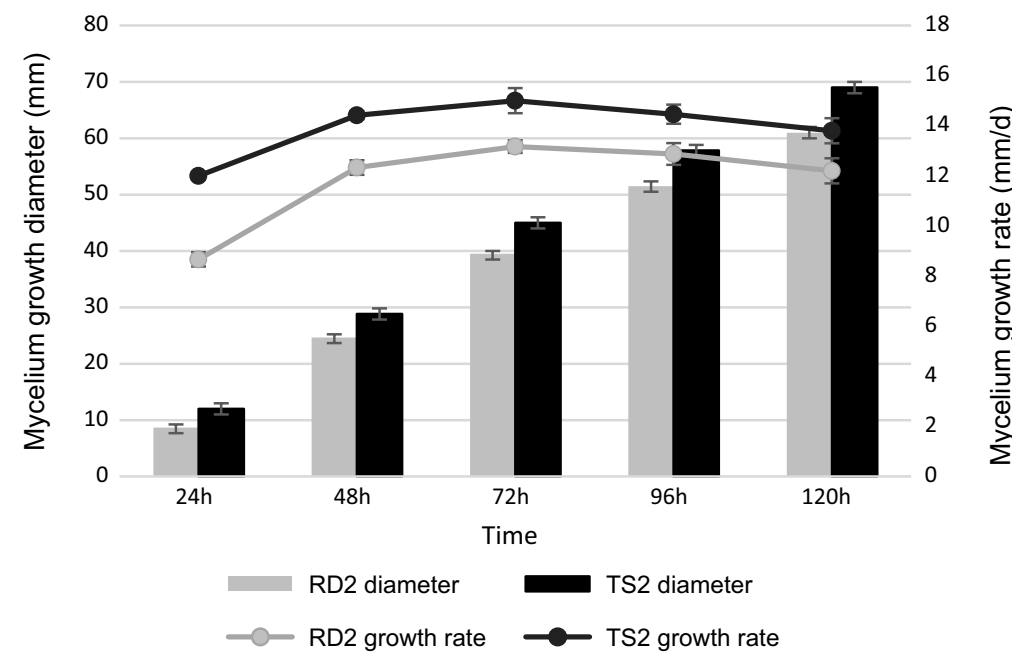

Nevertheless, the abnormal $\mathrm{pH}$ change from the ME to MS stage in TS2 koji might indicate different metabolic activities of TS2.

The amylase and protease activities of the two strains during koji fermentation were assayed and compared (Fig. 2c, d). Both strains displayed increasing enzyme activities along fermentation due to the accumulation of enzymes. However, RD2 showed a significantly higher protease activity than TS2, especially during the transition from the ES to MS stage, after which the protease activity of RD2 was threefold higher than that of TS2 (Fig. 2c). The lower protease activity in TS2 koji could directly result in a decreased amino acid yield and thus 
Fig. 2 Phenotypes of RD2 and TS2 during koji fermentation in three fermentation stages: growth (a), $\mathrm{pH}(\mathbf{b})$, amylolytic activities (c), and proteolytic activities (d). $M E$ mycelium expansion, $E S$ early sporulation, $M S$ mature sporulation. Data are presented as mean \pm standard deviation. ${ }^{*} P<0.05$ (Student's $t$ test) a
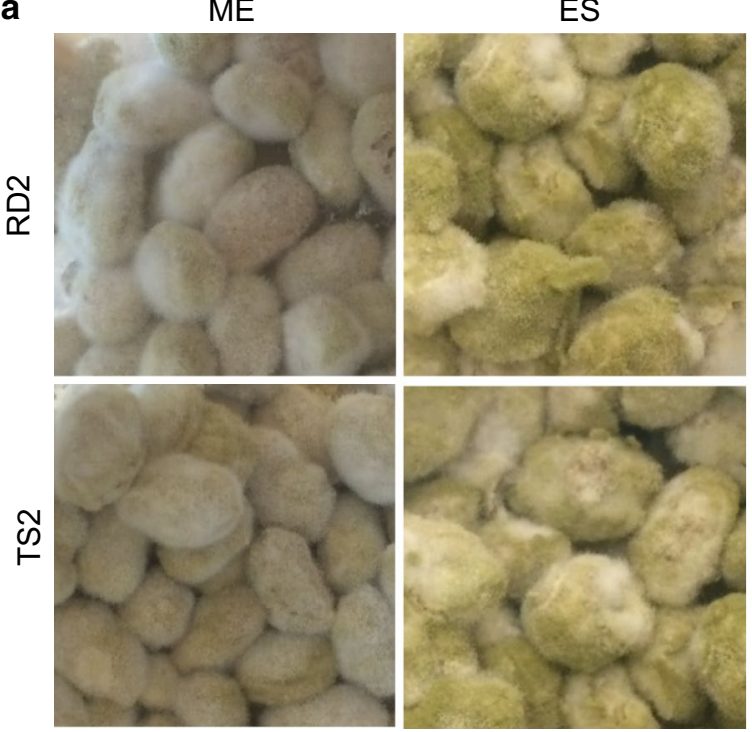

MS
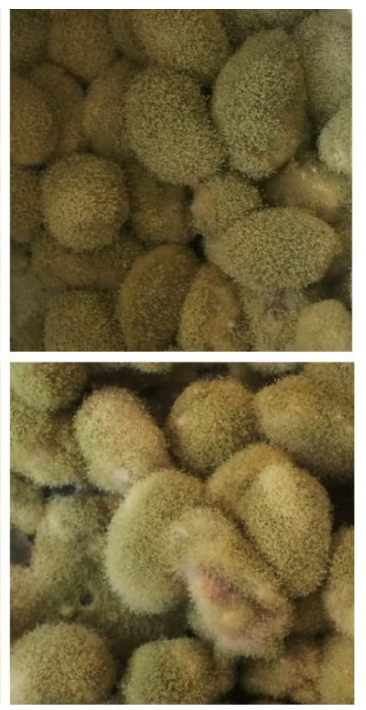

b
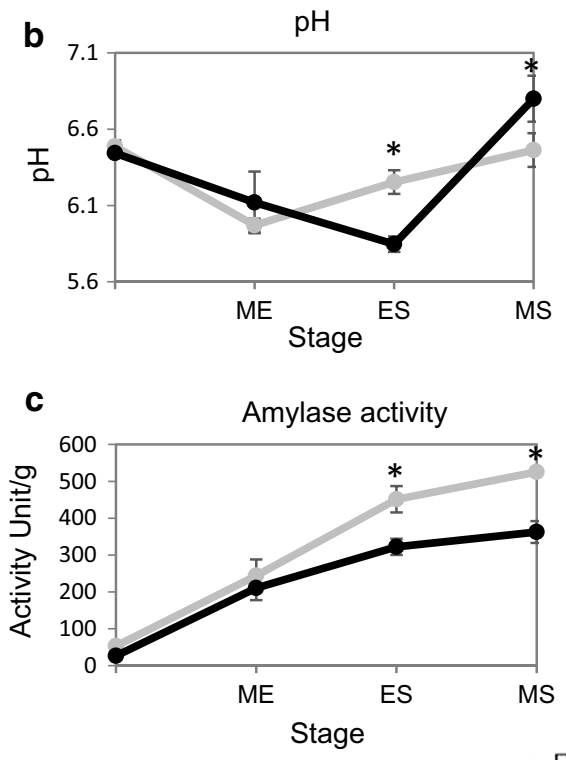

d

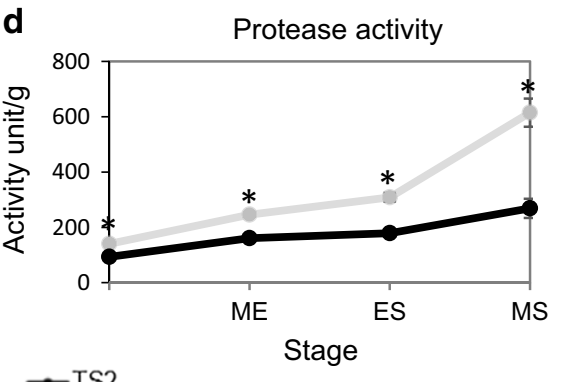
TS2

soy sauce with a poor quality. RD2 also displayed a significantly higher amylase activity than TS2 in the ES and MS stages (Fig. 2d), showing a stronger ability to utilize amylolytic carbohydrates in late koji fermentation. Noticeably, complete conidiation occurred synchronously with high enzyme activities in RD2, suggesting a correlation between conidiation and enzyme production. Indeed, te Biesebeke et al. [40] have proven that the production of amylases and proteases is positively related to the number of penetrating hyphae, which is the main apparatus to produce hydrolytic enzymes. Here, more spores in RD2 could geminate to grow more penetrating hyphae, which facilitated enzyme production. Thus, conidiation could reflect the level of enzyme production.

\section{General genomic statistics and variant calling}

DNA sequences of both strains were obtained by wholegenome sequencing using the Ion Torrent platform. General statistics of the genomes is summarized in Table 1. Aligning the reads to the reference RIB40 genome showed that highquality reads from both RD2 and TS2 covered over $95 \%$ of the reference genome with an average depth of $\sim 7 x$. Using SHRiMP2, 96.3\% of the RD2 reads and $95.2 \%$ of the TS2 reads were successfully mapped to the RIB40 genome. The internal transcribed spacer (ITS) and calmodulin sequences were recommended for the identification of aspergilli [36]. Sequences of both gene regions of TS2 showed $100 \%$ identity to those of RD2 and RIB40, excluding the possibility of contamination. Using the RD2 genome as reference, 858 
Table 1 General statistics of RD2 and TS2 genomes

\begin{tabular}{lll}
\hline & RD2 & TS2 \\
\hline Total number of bases & $318.08 \mathrm{Mbp}$ & $298.14 \mathrm{Mbp}$ \\
Total number of reads & $1,836,901$ & $1,706,630$ \\
Number of Q20 bases $^{\mathrm{a}}$ & $296.73 \mathrm{Mbp}$ & $279.25 \mathrm{Mbp}$ \\
Number of Q20 reads $^{\mathrm{b}}$ & $1,645,434$ & $1,538,563$ \\
Filtered reads $^{\mathrm{c}}$ & 191,467 & 168,067 \\
Mean read length & $172 \mathrm{bp}$ & $173 \mathrm{bp}$ \\
Longest read length & $366 \mathrm{bp}$ & $321 \mathrm{bp}$ \\
GC content & $47 \%$ & $46 \%$ \\
\hline
\end{tabular}

${ }^{a}$ Bases with quality $\geq 20$ are counted

${ }^{b}$ Reads with a base quality $\geq 20$ in more than half of the bases are counted

${ }^{c}$ Reads with a base quality $<20$ in more than half of the bases are filtered

SNPs and 50 indels were identified in TS2. The majority of SNVs ( $64.3 \%$ of SNPs, $74 \%$ of indels) occurred in the intergenic region. Excluding synonymous SNPs and mutation occurred in introns, 81 SNPs were predicted to cause 79 missense and two nonsense effects, and nine indels were predicted to cause frameshift effects, which could directly affect the structure and function of 66 genes (Table S3).

It is noteworthy that SNVs of TS2 were identified in genes related to hydrolysis of carbohydrates and amino acids. For instance, missense mutations were identified in beta-glucosidase (AO090001000266), alphaglucanase (AO090113000091) and carboxypeptidase $\mathrm{C}$ (AO090023000382); and a frameshift mutation was identified in tripeptidyl-peptidase (AO090701000907). These SNVs could result in malfunctional glycolytic and proteolytic enzymes and lower enzyme activities in TS2. SNPs found in two transcriptional factors (TFs) with predicted roles in carbohydrate metabolism regulation (AO090005001296, A0090103000131) could also influence the glycolytic activity of TS2. Interestingly, three missense mutations were identified in translation initiation factor 5A (elF-5A), which is specifically expressed in the penetrating hyphae to facilitate polypeptide synthesis during translation [29]. Since penetrating hyphae is the main apparatus to secrete hydrolytic enzymes [40], we speculate that TS2 is inferior in protein synthesis and thus enzyme secretion. Two mutated genes related to arginine metabolism are highlighted here: transmembrane arginine transporter (AO090023000039) and TF involved in arginine biosynthesis (AO090701000493). Arginine is a critical amino acid required for mycelium growth and conidiation, where a higher concentration is required for conidiation than mycelium growth [1]. The mutated arginine transporter and biosynthesis regulator could lower the concentration of arginine produced, leading to the conidiation defects in TS2.
TS2 also accumulated mutations in the metabolism of amino acids, fatty acids and isoprenoids, which can be precursors of flavor compounds. For instance, SNPs identified in cysteine synthase (AO090011000336) and glutathione S-transferase (AO090038000465) could hinder the enzymatic mediation of cysteine production, which would impair the formation of sulfur-containing flavors [38]. Indeed, protein metabolism and amino acid profiles have been suggested to play pivotal role in the determination of soy sauce flavor $[23,49]$. SNPs found in the potential regulator of amino acid biosynthesis (AO090020000331) and amino acid transporters (AO090026000734, AO090003000832) could therefore have prolonged negative effect on soy sauce flavor formation. Mutated genes related to fatty acid metabolism included lipase 2 precursor (AO090103000172), fatty acylCoA synthase (AO090001000249), and fatty acid synthase (AO090010000108). As fatty acids are precursors of predominant aroma in soy sauce [9], SNVs in lipid hydrolysis and fatty acid biosynthesis also explain the defects in flavor formation in TS2. Three missense mutations found in geranylgeranyl pyrophosphate synthase (AO090009000093) involved in the biosynthesis of isoprenoids may represent another evidence. Isoprenoids are aroma precursors identified in fermented soy sauce and soybean paste [13]. They can undergo intricate rearrangement and isomerization under acidic conditions to yield odor-active compounds such as methyl butanone derivatives, which have been detected in both koji and soy sauce $[9,38,49]$.

\section{Transcriptome comparison during koji fermentation}

To investigate the transcriptomic differences of RD2 and TS2 during koji fermentation, RNA extracted from three fermentation stages were subject to high-throughput Illumina sequencing. An average of 28,659,093 reads were obtained for each of the 12 samples (two strains in three stages, with duplicates). Over $90 \%$ raw reads could be mapped to the RIB40 genome with a tolerance of two mismatches (Table S1). A total of 1796, 1008 and 472 DEGs were identified in the ME, ES and MS stages, respectively. Functional analysis based on KOG revealed that DEGs up-regulated in RD2 were enriched in metabolism, especially transport and metabolism of carbohydrates, amino acids, lipids, and secondary metabolites; whereas TS2 possessed more up-regulated genes in information storage and processing, including DNA replication, transcription and translation (Fig. 3). Since RD2 yields better soy sauce when used in the koji fermentation, the differential expression profiles indicate key metabolism and transcriptional regulation that maintain the desirable koji fermentation performance. In addition, the number of DEGs decreased over time, indicating a stronger cellular response in early fermentation stage when nutrients were sufficient for 


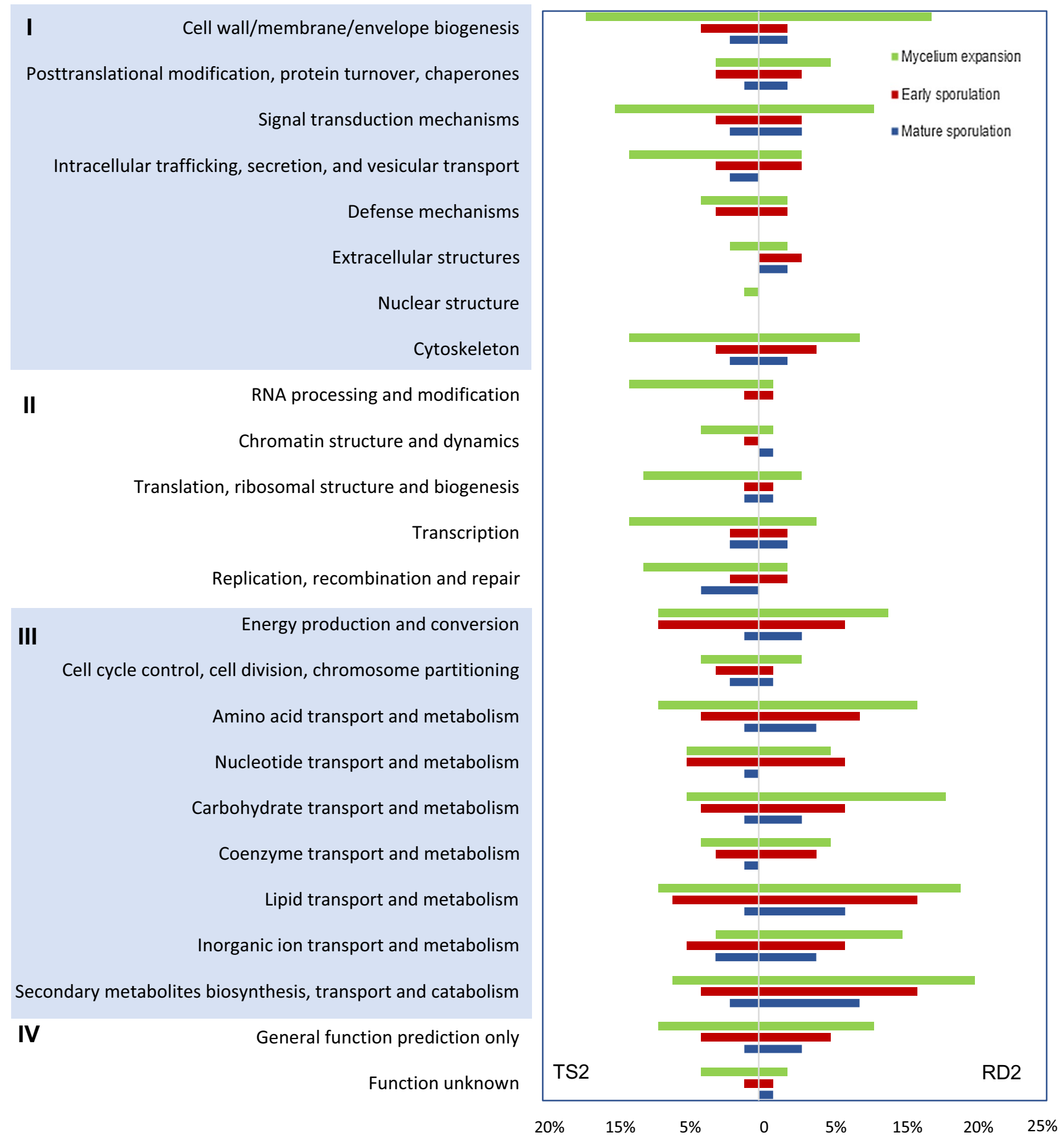

Fig. 3 EuKaryotic Orthologous Groups (KOG) analysis. Main categories: $I$ cellular processes and signaling, $I I$ information storage and processing, III metabolism, IV poorly characterized

growth and development. To verify the transcriptome data, six genes were selected for real-time RT-PCR (Table S2). The trends of up- and down-regulation of these genes were confirmed to be consistent with the RNA-Seq results (Fig. S1).

\section{Regulation of glycolytic and proteolytic enzyme production}

The ability to produce hydrolytic enzymes by A. oryzae is of great concern when considering the performance of 
koji fermentation. To investigate the expression of glycolytic and proteolytic enzymes during koji fermentation, we analyzed the identified DEGs based on the carbohydrate-active enzyme database (http://www.cazy.org/) [24] and MEROPS peptidase database (https://www.ebi.ac.uk/ merops) [35]. Results revealed that 101 and 46 DEGs were involved in the hydrolysis of carbohydrates and proteins, respectively (Fig. 4), including both well-curated and in silico predicted hydrolytic enzymes. In general, RD2 possessed more up-regulated genes encoding hydrolytic enzymes than TS2, supporting the higher enzyme activities observed in RD2. We thereby provided a completed expression profile of important hydrolytic enzymes that are necessary to maintain koji fermentation performance.

Besides the well-known amylolytic enzymes that are highly expressed in soy sauce-producing strains [22, 28, 43], various cellulolytic, xylanolytic and pectinolytic enzymes were also expressed in significantly higher levels in RD2 in the ME and ES stages (Fig. 4a). Among these, alpha-galactosidases, up-regulated in RD2 in all three stages, can effectively degrade the soybean sugars-raffinose and stachyose. Since these enzymes require corresponding carbohydrates for inducement [14], our results suggest a better hyphal penetration of RD2 into soybeans, which contain the most plant polysaccharides including cellulose, pectin, raffinose, and stachyose within the substrates [8]. A network of TFs has been reported to regulate the expression of these enzymes [19]. Here, three up-regulated TFs in RD2 might be highly related: A0090020000656, a homolog of clrA in A. nidulans that functions as the activator of cellulases and xylanases [6]; manR, an activator of beta-glucosidases and alpha-galactosidases in the degradation of cellulose, hemicellulose, raffinose, and stachyose [32]; and A0090005000121, a homolog of rhaR in A. niger that acts as the activator of rhamnogalacturonan hydrolase and pectin lyases involved in pectin degradation [12].

By contrast, few plant polysaccharide-degrading enzymes were induced in TS2, since there was no up-regulation of the aforementioned regulators and the hyphal penetration was limited. However, there was an up-regulation of glucose-release enzymes including maltase and trehalase in the ES and MS stages of TS2, suggesting that this strain exhaustedly exploited preferred carbon source maltose for proliferation. In fact, it is less energy-intensive and more cost-efficient to directly use preferred carbon source than secrete plant polysaccharide-degrading enzymes for alternative carbon sources [19]. This potential enables a fast growth of TS2 as observed in Figs. 1a, 2a. On the other hand, active metabolism of various polysaccharides in RD2 (Fig. S2) ensures a simple sugar reserve for subsequent fermentation and facilitates the utilization of proteins as plant polysaccharide-degrading enzymes are coordinately responsible for the degradation of plant cell walls and the solubilization of proteins $[17,19]$.

Production of protease is a major concern in soy sauce koji fermentation since it directly relates to the amino acid yield and thus product quality. Here, RD2 possessed highly up-regulated peptidases including acid proteases aspergillopepsins, neutral proteases and alkaline protease oryzin in all three stages (Fig. 4b). This result is consistent with the high protease yield reported in other soy sauce-producing strains $[43,50]$. The largest fold change (up to 18 -fold) was observed in aspergillopepsin II (A0090023000393), which was barely expressed by TS2, especially in the MS stage. Other acid proteases including serine-type carboxypeptidase (AO090012000706), tripeptidase A (tppA) and aspergillopepsin $\mathrm{O}($ реp$O)$ were also expressed in extremely low levels in TS2 in the MS stage. Here, $\mathrm{pH}$ could act as a regulator because $p a c C$ was expressed at an about fivefold higher level in TS2 in the MS stage, at which the $\mathrm{pH}$ of koji experienced a dramatic increase (Fig. 2b). PacC is active in alkaline conditions and can repress acid-expressed genes and activate alkaline-expressed genes in $A$. nidulans, or function only as a repressor of acid-expressed genes in A. niger or Saccharomyces cerevisiae [34]. It can specifically bind to its target genes with the binding sequence of 5'-GCCARG-3', which is present in the promoter region of AO090012000706, tppA, pepO $O$ at the $-242,-290,-308$ positions, respectively. A. oryzae grows in a slightly acidic condition during koji fermentation, but it may suffer from stress in response to rapid alkalization [34], as shown in TS2 (Fig. 2b). Therefore, highly induced $p a c C$ in TS2 probably represses the expression of acidic proteases in late fermentation stage. A higher production of acidic proteases has been reported to be advantageous in substrate utilization and product quality improvement $[48,50]$. Thus, it is possible to improve koji fermentation performance by monitoring $\mathrm{pH}$ or manipulating $\mathrm{pH}$-related TFs like pacC. It is noteworthy that alkaline proteases leucine aminopeptidase and oryzin, and neutral proteases I and II were also expressed in extremely low levels in TS2, especially in the MS stage, leading to lower protease activity in late fermentation stage. te Biesebeke et al. has suggested the induction of pacC on oryzin and neutral proteases [41]. However, no inductive effect was observed upon the up-regulation of $\mathrm{pacC}$ in TS2 here.

Some other regulatory mechanisms may also be responsible for the overall enhanced protease production in RD2. As aforementioned, conidiation can be linked to enzyme production. In fact, protease production of $A$. oryzae has been proven to be closely related to conidiation [3]. A possible explanation is that some TFs positively regulating conidiation also play inductive roles in protease production [31, 39]. One example is the sporulation activator $f b C$, as $f l b C$ disrupted mutant shows significantly decreased expression of acid protease pepO and glucoamylase glaB [39]. Here, 


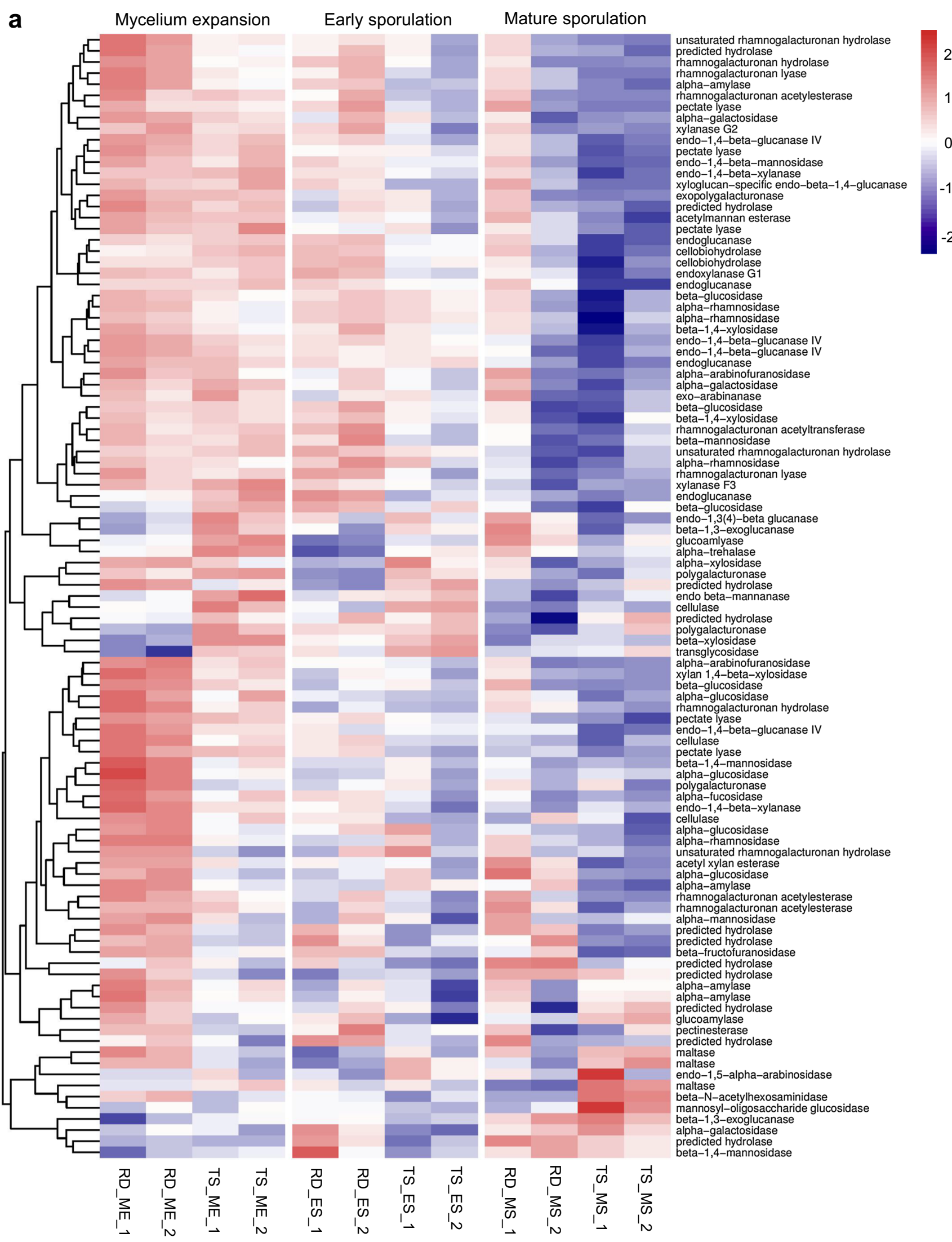

Fig. 4 Heatmaps from hierarchical clustering of differentially expressed genes encoding degrading enzymes of carbohydrates (a) and proteins (b) 


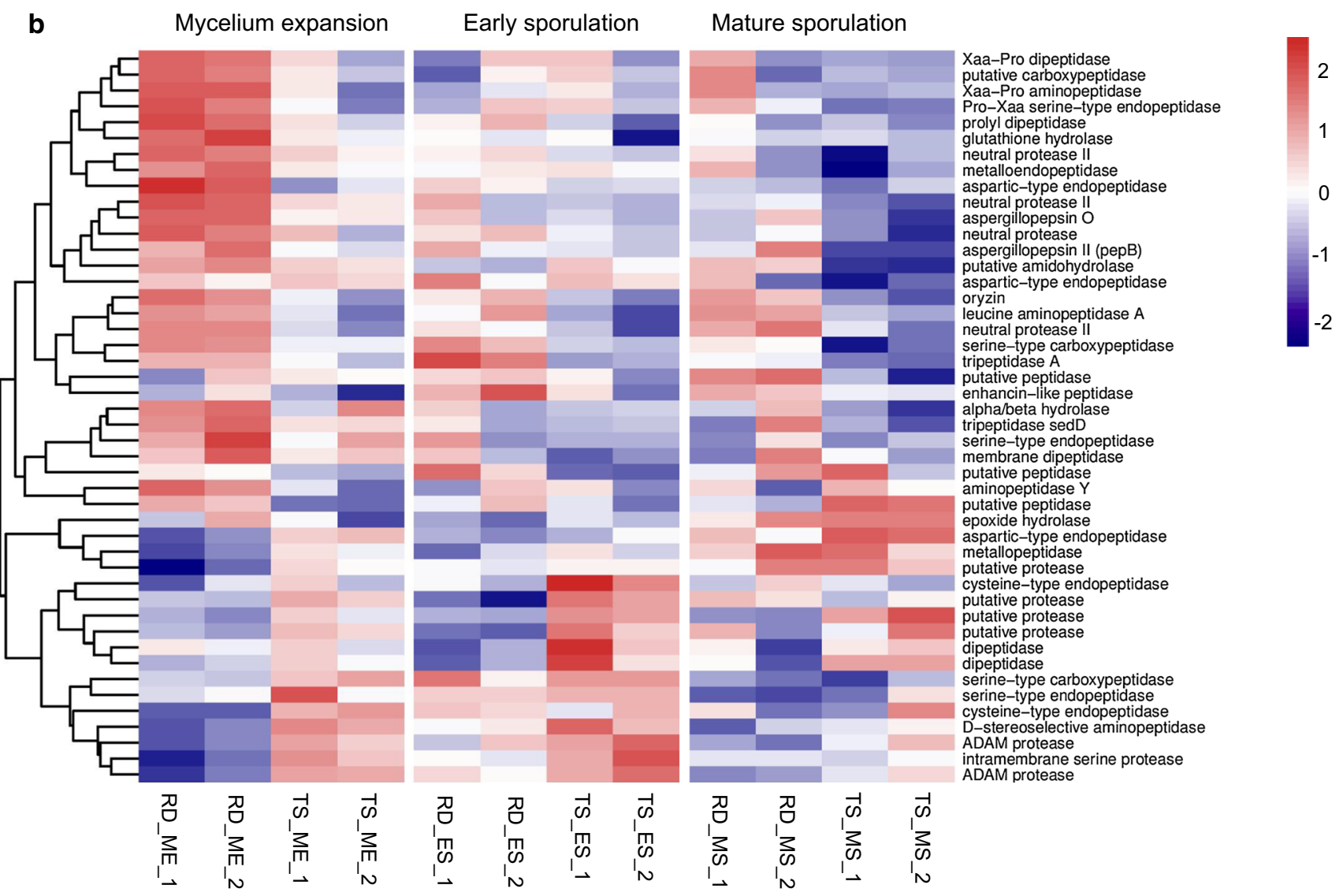

Fig. 4 (continued)

we found that three regulators of conidiation, $A b C$, atfB and AO090003001259, were up-regulated in RD2 in one or more stages. Although the exact mechanism is unknown, these regulators likely contribute to the increased protease production in RD2. In addition, the better hyphal penetration due to up-regulated plant polysaccharide-degrading enzymes in RD2 may allow better exposure to proteins inside soybeans, which can also contribute to protease production [3].

\section{Regulation of central carbon metabolism on koji fermentation performance}

One of the most striking findings of this study is the distinct gene expression profiles of central carbon metabolism between the two strains (Fig. 5, S3). In the ES stage, glycolysis and TCA cycle in TS2 were highly induced (Fig. 5, Table 2). However, in RD2, pentose phosphate pathway was induced. These results indicate different fates of glucose in the two strains.

In TS2, glucose is first channeled to phosphoenolpyruvate through up-regulated $f p b A, g p d A, p g k A$, and enoA, thereby generating more pyruvate, which then entails a large cascade of TCA cycle reactions involving up-regulated AO090023000801, $m d h A$ and $s d h B, s d h C$. The up-regulated TCA cycle in TS2 possibly supports the pH decrease in the ES stage (Fig. 2b) because acids produced via the TCA cycle, such as succinate and citrate, are the major reason of acidification during koji fermentation [33]. Apart from the larger amount of pyruvate generated by glycolysis, the up-regulated aspartate transaminase and serine/threonine ammonia-lyase involved in the catabolism of glutamate, aspartate, cysteine, tyrosine, and serine to pyruvate and TCA metabolites can also feed the TCA cycle in TS2. Thus, more ATPs can be generated via the TCA cycle in TS2, providing sufficient energy to support the vegetative growth of multicellular microorganisms [4], which explains the fast growth of TS2. By contrast, reduced expression of genes involved in glycolysis and TCA cycle and increased expression of those involved in hydrolytic enzyme production were observed in RD2. A low expression of glucose catabolic genes could release catabolite repression, contributing to the elevated level of hydrolytic enzyme production [28]. Therefore, glycolysis and TCA cycle may play a contradictive role in hydrolytic enzyme production, and highly induced glycolysis and TCA cycle in TS2 is unfavorable during koji fermentation. 


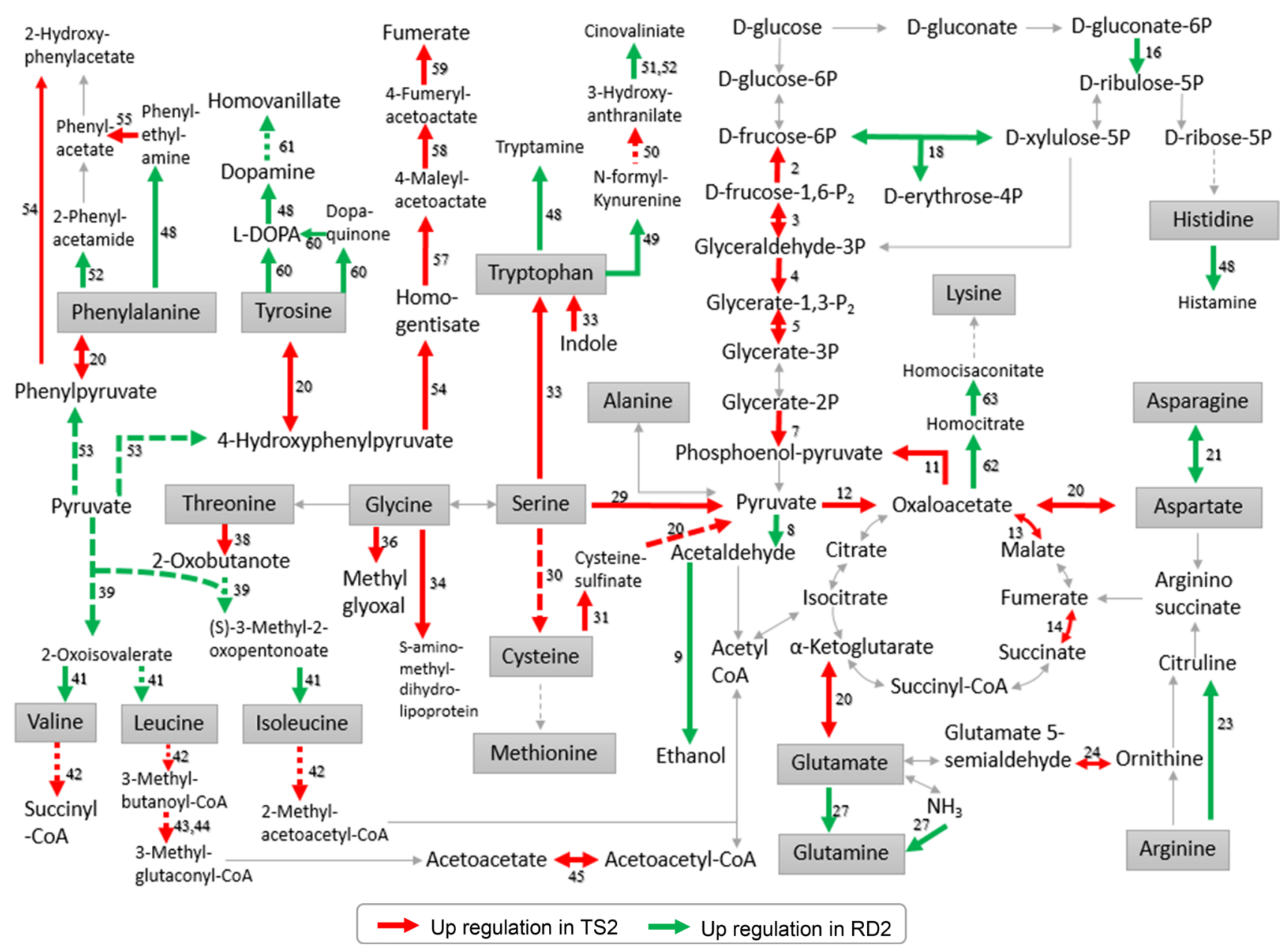

Fig. 5 Simplified KEGG pathways showing differentially expressed genes in the early sporulation stage that were mapped on the central carbon metabolism and related amino acid metabolism. Red and green arrows indicate up-regulated reactions in TS2 and RD2,

In RD2, up-regulated A0090003000121 and A0090012000526 direct the glucose metabolism to the pentose phosphate pathway (Fig. 5). The pentose phosphate pathway generates xylulose-5-phosphate, ribulose-5-phosphate and ribose-5-phosphate, which are precursors of characteristic flavor compounds in soy sauce such as 4-hydroxy-2(or 5)-ethyl5(or 2)-methyl-3(2H)-furanone (HEMF), guanosine-5'monophosphate (GMP) and inosine-5'-monophosphate (IMP) [38]. It is evident that the metabolic fate of glucose in RD2 is channeled to flavor precursor accumulation for better koji fermentation. Besides, genes involved in umami-flavored glutamate biosynthesis, including glutaminase and glutamate dehydrogenase, were up-regulated in the late fermentation stage. Branched-chain amino acid transaminase was also up-regulated in the ES and MS stages for valine, leucine and isoleucine biosynthesis, which are precursors of aroma compounds such as 2-methyl-propanal, 2-methyl-butanal and 3-methyl-butanal [9, 49]. Genes involved in the catabolism of aromatic amino acids respectively; dashed arrows indicate more than one step of reaction involved. DEG numbers correspond to the enzymes listed in Table 2. $P$ phosphate, $L$-DOPA 3,4-dihydroxy-L-phenylalanine

tyrosine (melO), phenylalanine (AO090038000057) and tryptophan (AO090038000578) were induced, where the related catabolites can contribute to flavor formation [9]. By contrast, multiple amino acids were converted to energy for fast growth in TS2, hindering the production of flavor precursors necessary in soy sauce flavor formation.

Here, different survival strategies are utilized by RD2 and TS2: TS2 exploits glucose efficiently and burns extra amino acids to fuel glycolysis and TCA cycle for mycelium growth, making it a superior strain in survival competition, whereas RD2 sacrifices mycelium growth for elevated hydrolytic enzyme production and emphasizes metabolic pathways of flavor precursor formation, making it a favorable strain in koji fermentation. Upon the efficiency of nutrient utilization, TS2 is a superior strain with a higher adaptability to the koji fermentation environment. Since fast growth via induced glycolytic catabolism has extensive impact on both hydrolytic 
Table 2 Expression fold change of differentially expressed genes (DEGs) involved in central carbon metabolism and related metabolism

\begin{tabular}{|c|c|c|c|c|c|c|}
\hline \multirow[t]{2}{*}{ Number } & \multirow[t]{2}{*}{ Gene ID } & \multirow[t]{2}{*}{ EC number } & \multirow[t]{2}{*}{ Gene function } & \multicolumn{3}{|c|}{$\log _{2}(\text { fold change })^{\mathrm{a}}$} \\
\hline & & & & $\mathrm{ME}$ & ES & MS \\
\hline 1 & $p f k A$ & 2.7.1.11 & 6-Phosphofructokinase & 1.09 & -0.36 & -0.56 \\
\hline 2 & $f b p A$ & 3.1.3.11 & Fructose-1,6-bisphosphatase & -0.06 & 1.58 & -0.68 \\
\hline 3 & $f b a A$ & 4.1.2.13 & Fructose-1,6-bisphosphate aldolase & 0.05 & 1.43 & -0.25 \\
\hline 4 & gpdA & 1.2.1.12 & Glyceraldehyde-3-phosphate dehydrogenase & -0.13 & 1.86 & -0.74 \\
\hline 5 & $p g k A$ & 2.7 .2 .3 & 3-Phosphoglycerate kinase & 0.09 & 2.08 & -0.86 \\
\hline 6 & gртA & 5.4.2.12 & Phosphoglyceromutase & 1.16 & 0.54 & -0.23 \\
\hline 7 & enoA & 4.2.1.11 & Enolase & 0.13 & 1.60 & -0.24 \\
\hline 8 & A0090003000661 & 4.1.1.1 & Pyruvate decarboxylase & -0.31 & -1.64 & -1.50 \\
\hline 9 & AO090003000261 & 1.1.1.1 & Alcohol dehydrogenase & -2.45 & -1.29 & -2.33 \\
\hline 10 & AO090023000467 & 1.2.1.3 & Aldehyde dehydrogenase & -1.03 & -0.85 & 0.14 \\
\hline 10 & AO090026000741 & 1.2.1.5 & Aldehyde dehydrogenase & -1.13 & 0.19 & -0.80 \\
\hline 11 & A0090003000174 & 4.1.1.49 & Phosphoenolpyruvate carboxykinase & 1.90 & 4.62 & -0.96 \\
\hline 12 & A0090023000801 & 6.4.1.1 & Pyruvate carboxylase & -0.16 & 1.39 & -0.51 \\
\hline 13 & $m d h A$ & 1.1.1.37 & Malate dehydrogenase & 0.21 & 1.55 & 0.19 \\
\hline 14 & $s d h B$ & 1.3.5.1 & Succinate dehydrogenase & -0.15 & 1.38 & -0.43 \\
\hline 14 & $s d h C$ & 1.3.5.1 & Succinate dehydrogenase & -0.23 & 1.06 & -0.33 \\
\hline 15 & A0090003001003 & 2.7.1.12 & Gluconokinase & 1.24 & -0.41 & -0.72 \\
\hline 16 & AO090003000121 & 1.1.1.44 & 6-Phosphogluconate dehydrogenase & 0.11 & -1.52 & -0.09 \\
\hline 17 & A0090026000544 & 5.3.1.6 & Ribose-5-phosphate isomerase A & -1.51 & -0.26 & -0.36 \\
\hline 18 & AO090012000526 & 2.2.1.1 & Transketolase & -0.02 & -1.19 & 0.12 \\
\hline 19 & A0090003000625 & 4.1.2.9 & Fructose-6-phosphate phosphoketolase & 1.81 & 0.93 & -1.85 \\
\hline 20 & A0090003001171 & 2.6.1.1 & Aspartate transaminase & -0.10 & 1.25 & -1.23 \\
\hline 21 & A0090120000180 & 6.3.5.4 & Asparagine synthase & -1.21 & -1.38 & 0.53 \\
\hline 22 & A0090023000395 & 6.3.4.5 & Argininosuccinate synthase & -1.35 & -0.71 & -0.78 \\
\hline 23 & A0090138000171 & 1.14 .13 .39 & Nitric oxide synthase & -1.09 & -1.46 & 0.22 \\
\hline 24 & A0090023000546 & 2.6.1.13 & Ornithine-oxo-acid transaminase & -0.03 & 1.82 & 0.60 \\
\hline 25 & A0090001000549 & 1.2.1.88 & 1-Pyrroline-5-carboxylate dehydrogenase & -2.42 & 0.21 & -0.50 \\
\hline 26 & A0090005000539 & 4.1.1.15 & Glutamate decarboxylase & 1.16 & -0.32 & -0.52 \\
\hline 27 & A0090011000308 & 6.3 .1 .2 & Glutamine synthetase & 2.69 & -2.98 & 0.86 \\
\hline 28 & gtaA & 3.5.1.2 & Glutaminase & -0.16 & -0.19 & -1.54 \\
\hline 29 & AO090023000790 & 4.3.1.17 & Serine/threonine amonialyase & 0.92 & 3.19 & -0.17 \\
\hline 30 & A0090102000630 & 2.5.1.47 & Cysteine synthase A & -0.10 & 1.29 & -0.83 \\
\hline 31 & AO090020000659 & 1.13 .11 .20 & Cysteine dioxygenase & -2.74 & 3.25 & -2.08 \\
\hline 32 & A0090038000357 & 2.5.1.6 & $S$-Adenosylmethionine synthetase & -0.02 & -0.11 & 1.05 \\
\hline 33 & A0090023000874 & 4.2.1.20 & Tryptophan synthase & -1.11 & 1.22 & 0.46 \\
\hline 34 & A0090011000351 & 1.4.4.2 & Glycine dehydrogenase & 0.41 & 1.62 & 0.21 \\
\hline 35 & A0090005000497 & 4.1.2.48 & Threonine aldolase & 1.46 & 0.25 & 1.31 \\
\hline 36 & A0090005000103 & 1.4.3.21 & Primary amine oxidase & -2.21 & 1.25 & 0.15 \\
\hline 37 & A0090005000038 & 1.5.3.1 & Sarcocine oxidase & -1.15 & -0.76 & -0.70 \\
\hline 38 & AO090023000790 & 4.3.1.19 & Threonine dehydratase & 0.92 & 3.19 & -0.17 \\
\hline 39 & AO090166000076 & 2.2.1.6 & Acetolactate synthase & 1.55 & -1.07 & 1.32 \\
\hline 40 & A0090009000414 & 4.2.1.9 & Dihydroxy acid dehydratase & -1.43 & -0.18 & -1.47 \\
\hline 41 & A0090005000936 & 2.6.1.42 & Branched-chain amino acid transaminase & -0.56 & -1.79 & -0.81 \\
\hline 41 & A0090011000044 & 2.6.1.42 & Branched-chain amino acid transaminase & -0.32 & 0.44 & -1.31 \\
\hline 42 & AO090001000555 & 1.2.4.4 & $\begin{array}{l}\text { Branched-chain amino acid alpha-keto acid dehy- } \\
\text { drogenase }\end{array}$ & -1.58 & 2.40 & -1.03 \\
\hline 43 & $i v d A$ & 1.3.8.4 & Isovaleryl-CoA dehydrogenase & -1.58 & 1.66 & -0.35 \\
\hline 44 & A0090020000492 & 6.4.1.4 & 3-Methylcrotonyl-coA carboxylase & -1.35 & 1.35 & -0.12 \\
\hline
\end{tabular}


Table 2 (continued)

\begin{tabular}{|c|c|c|c|c|c|c|}
\hline \multirow[t]{2}{*}{ Number } & \multirow[t]{2}{*}{ Gene ID } & \multirow[t]{2}{*}{ EC number } & \multirow[t]{2}{*}{ Gene function } & \multicolumn{3}{|c|}{$\log _{2}(\text { fold change })^{\mathrm{a}}$} \\
\hline & & & & ME & ES & MS \\
\hline 45 & A0090009000195 & 2.8.3.5 & 3-Oxoacid CoA-transferase & -1.37 & 1.12 & -1.28 \\
\hline 46 & A0090103000406 & 2.3.1.9 & Acetyl-CoA acetyltransferase & -1.43 & 0.87 & -1.00 \\
\hline 47 & AO090023000518 & 1.1.1.31 & 3-Hydroxyisobutyrate dehydrogenase & -0.72 & -0.36 & -1.08 \\
\hline 48 & A0090038000057 & 4.1.1.28 & Aromatic amino acid decarboxylase & -1.23 & -1.07 & 0.35 \\
\hline 49 & A0090038000578 & 1.13 .11 .52 & Indoleamine 2,3-dioxygenase & -1.38 & -1.97 & 0.06 \\
\hline 50 & A0090003001247 & 3.7.1.3 & Kynureninase & -0.79 & 3.97 & -1.96 \\
\hline 51 & catA & 1.11.1.6 & Catalase & 0.53 & -1.23 & -0.61 \\
\hline 51 & catB & 1.11.1.6 & Catalase & -1.49 & -0.43 & -0.90 \\
\hline 52 & AO090010000722 & 1.11.1.21 & Catalase peroxidase & 0.50 & -1.90 & 2.20 \\
\hline 53 & A0090005000086 & 2.5 .1 .54 & 3-Deoxy-7-phosphoheptulonate synthase & -0.15 & -1.15 & -0.77 \\
\hline 54 & A0090003000208 & 1.13.11.27 & 4-Hydroxyphenylpyruvate dioxygenase & -1.35 & 6.25 & -2.99 \\
\hline 55 & A0090005000103 & 1.4.3.21 & Copper amine oxidase & -2.21 & 1.25 & 0.15 \\
\hline 56 & A0090003001361 & 1.14.14.54 & Phenylacetate 2-hydroxylase & -2.25 & -0.33 & -1.36 \\
\hline 57 & A0090003000210 & 1.13 .1 .15 & Homogentisate 1,2-dioxygenase & -1.47 & 4.26 & -2.00 \\
\hline 58 & AO090003000212 & 5.2 .1 .2 & Maleylacetoacetate isomerase & -0.49 & 3.00 & -1.48 \\
\hline 59 & A0090003000211 & 3.7.1.2 & Fumarylacetoacetase & -1.18 & 3.22 & -0.98 \\
\hline 60 & melO & 1.14.18.1 & Tyrosinase & -1.63 & -2.82 & -1.41 \\
\hline 61 & A0090701000285 & 2.1.1.6 & Catechol- $O$-methyltransferase & 0.22 & -1.83 & 0.73 \\
\hline 62 & AO090003001165 & 2.3.3.14 & Homocitrate synthase & 0.56 & -1.19 & 0.01 \\
\hline 63 & A0090001000484 & 4.2.1.- & Homoaconitase & 0.07 & -1.10 & -0.26 \\
\hline 64 & A0090001000233 & 1.5.1.10 & Saccharopine dehydrogenase & 1.53 & -0.62 & -0.62 \\
\hline 65 & AO0990012000450 & 2.6.1.9 & Histidinol phosphate aminotransferase & 0.05 & 0.07 & 1.05 \\
\hline
\end{tabular}

${ }^{\mathrm{a}} \log _{2}$ (fold change) $<-1$ indicates the up-regulation in $\mathrm{RD} 2, \log _{2}$ (fold change) $>1$ indicates the up-regulation in TS2

enzyme production and flavor precursor formation, the control of glycolysis and TCA cycle at the transcriptional level represents a possible solution. The gene $A Z F 1$ has been reported to extensively induce the glycolytic catabolic pathways in S. cerevisiae [37]. Here, homologs of AZF 1 (AO090003001179) was found up-regulated in TS2 in the ME stage, which may be related to the regulation of glycolysis and TCA cycle. Also, a set of up-regulated TFs of mycelium growth that may influence glycolysis and TCA cycle in TS2 were identified here: homologs of ASG1 (AO090003001246, AO090009000029), homologs of ECM22 (AO090023000416, AO090010000097) and UPC2 (AO090009000133, AO090010000546), which have redundant role in up-regulation of filamentous growth in S. cerevisiae [27, 46]. Of these, AO090023000416 was upregulated at about fivefold in TS2 in the three fermentation stages, possibly supporting the overall faster growth but repressing the production of hydrolytic enzymes and flavor precursor formation. The exact relationship between mycelium growth and fermentation performance with certain involvement of regulators need to be tested and discussed in future studies.

\section{Regulation on the formation of other flavor precursors}

RD2 showed a stronger metabolic capacity of fatty acid, which are important flavor precursors in soy sauce [7]. For instance, triglyceride lipases (AO090012000352, A0090001000143, A0090701000542), fatty acid synthase (fasl, AO090011000046), aldehyde dehydrogenase (AO090023000467), and alcohol dehydrogenases (A0090003000261, A0090003001407) were up-regulated in RD2. The up-regulation of these enzymes largely induced the accumulation of fatty acids, which can be further catabolized by up-regulated aldehyde dehydrogenases and alcohol dehydrogenases to important aroma compounds, such as (E)-2-octenal, 1-octen-3-one, 5-methyl3-heptanone, 3-octanol, and 1-octen-3-ol, which have been detected in mature koji $[9,38,49]$. Three up-regulated TFs (AO090005000336, AO090001000029, A0090023000362) with a predicted role in fatty acid catabolism may be responsible for the induction of aldehyde dehydrogenase and alcohol dehydrogenase. Moreover, this process is believed to be related to the sporulation of $A$. oryzae [16]. Here, better conidiation observed in RD2 probably supports the enhanced fatty acid metabolism. 
Secondary metabolite isoprenoids can contribute to the overall aroma of fermented soy sauce [13]. Tetraterpenoid known as carotenoid can be readily catalyzed to various odor-active 2-butanone derivatives detected in soy sauce koji $[9,30,38]$. Here, acetyl-CoA acetyltransferase (AO090103000406) and isopentenyl-diphosphate Deltaisomerase (AO090023000500) that can induce the elongation of isoprenoid backbone and phytoene dehydrogenase (AO090020000158) used in the biosynthesis of carotenoid were up-regulated in RD2. The corresponding regulator hydroxymethylglutaryl-CoA reductase (AO090701000640) [45] was up-regulated in RD2, which may be responsible for the induced isoprenoid metabolism.

\section{Conclusions}

In this study, we applied comparative genomics to examine genome variants in the genome of TS2 compared to RD2, and performed comparative transcriptomics to investigate differential gene expression patterns between the two strains in three stages of soy sauce koji fermentation. Our results showed that the phenotypic characteristics of TS2 could be explained by the genotypic defects observed-SNVs were found in genes related to the production of hydrolytic enzymes and formation of flavor precursors, indicating the importance of massive and intact reserve of hydrolytic enzymes and flavor precursors in ideal koji fermentation. The mutated gene loci may therefore represent molecular markers for koji fermentation performance. Transcriptomic analysis has confirmed the advantages of RD2 in hydrolytic enzyme production and flavor precursor formation, and rationalized corresponding regulation mechanisms involving fermentation parameters such as $\mathrm{pH}$ and conidiation of $A$. oryzae via identification of a set of TFs. The growth advantage of TS2 probably results from enhanced central carbon metabolism and amino acid catabolism, which in turn extensively lower the fermentation performance, suggesting the necessity of manipulating these metabolic pathways for desirable fermentation. By integrated comparative genomes and transcriptomes, a list of potential molecular markers related to desirable koji fermentation was identified here. This allows future evaluation of the fermentation capacity of $A$. oryzae strains by monitoring the gene structure, production level and activity of targeted proteins. Our study has therefore provided insights into targeted strain maintenance and strain improvement for a better fermentation process and soy sauce quality.

Open Access This article is distributed under the terms of the Creative Commons Attribution 4.0 International License (http://creativeco mmons.org/licenses/by/4.0/), which permits unrestricted use, distribution, and reproduction in any medium, provided you give appropriate credit to the original author(s) and the source, provide a link to the Creative Commons license, and indicate if changes were made.

\section{References}

1. Adams TH, Wieser JK, Yu JH (1998) Asexual sporulation in Aspergillus nidulans. Microbiol Mol Biol Rev 62:35-54

2. Andrew S (2010) FastQC: a quality control tool for high throughput sequence data. Babraham Bioinfom http://www. bioinformatics.babraham.ac.uk/projects/fastqc. Accessed 17 Oct 2016

3. Battaglino RA, Huergo M, Pilosof AMR, Bartholomai GB (1991) Culture requirements for the production of protease by Aspergillus oryzae in solid state fermentation. Appl Microbiol Biotechnol 35:292-296

4. Chambergo FS, Bonaccorsi ED, Ferreira AJ, Ramos AS, Ferreira Júnior JR, Abrahao-Neto J, Farah JP, El-Dorry H (2002) Elucidation of the metabolic fate of glucose in the filamentous fungus Trichoderma reesei using expressed sequence tag (EST) analysis and cDNA microarrays. J Biol Chem 277:13983-13988

5. Cingolani P, Platts A, Wang LL, Coon M, Nguyen T, Wang L, Land SJ, Lu X, Ruden DM (2012) A program for annotating and predicting the effects of single nucleotide polymorphisms, SNPeff: SNPs in the genome of Drosophila melanogaster strain w1118; iso-2; iso-3. Fly 6:80-92

6. Coradetti ST, Craig JP, Xiong Y, Shock T, Tian C, Glass NL (2012) Conserved and essential transcription factors for cellulase gene expression in ascomycete fungi. Proc Natl Acad Sci 109:7397-7402

7. David M, Dzamba M, Lister D, Ilie L, Brudno M (2011) SHRiMP2: sensitive yet practical short read mapping. Bioinformatics 27:1011-1012

8. Eldridge AC, Black LT, Wolf WJ (1979) Carbohydrate composition of soybean flours, protein concentrates, and isolates. J Agric Food Chem 27:799-802

9. Feng Y, Cui C, Zhao H, Gao X, Zhao M, Sun W (2013) Effect of koji fermentation on generation of volatile compounds in soy sauce production. Int J Food Sci Technol 48:609-619

10. Fuwa H (1954) A new method of microdetermination of amylase activity by the use of amylase as the substrate. J Biochem 41:583-603

11. Gomi K, Abe K (2008) Food products fermented by Aspergillus oryzae. In: Goldman GH, Osmani SA (eds) The Aspergilli: genomics, medical aspects, biotechnology, and research methods. CRC Press, Boca Raton, pp 427-437

12. Gruben BS, Zhou M, Wiebenga A, Ballering J, Overkamp KM, Punt PJ, de Vries RP (2014) Aspergillus niger RhaR, a regulator involved in L-rhamnose release and catabolism. Appl Microbiol Biotechnol 98:5531-5540

13. Huang TC, Teng DF (2004) Soy sauce: manufacturing and biochemical changes. In: Hui YH, Meunier-Goddik L, Josephsen J, Nip WK, Stanfield PS (eds) Handbook of food and beverage fermentation technology, vol 134. CRC Press, Boca Raton, pp 497-532

14. Huberman LB, Liu J, Qin L, Glass NL (2016) Regulation of the lignocellulolytic response in filamentous fungi. Fungal Biol Rev 30:101-111

15. Jin FJ, Takahashi T, Matsushima K, Hara S, Shinohara Y, Maruyama J, Ketamoto K, Koyama Y (2011) SclR, a basic helix-loophelix transcription factor, regulates hyphal morphology and promotes sclerotial formation in Aspergillus oryzae. Eukaryot Cell 10:945-955

16. Kaminski E, Stawcki S, Wasowicz E (1974) Volatile flavour compounds produced by molds of Aspergillus, Penicillium, and fungi imperfecti. Appl Environ Microbiol 27:1001-1004

17. Kobayashi T, Abe K, Asai K, Gomi K, Juvvadi PR, Kato M, Kitamoto K, Takeuchi M, Machida M (2007) Genomics of Aspergillus oryzae. Biosci Biotechnol Biochem 71:646-670 
18. Kolde R (2015) Pheatmap: pretty heatmaps. R package version 1.0.8. https://cran.r-project.org/web/packages/pheatmap/pheat map.pdf. Accessed 15 Aug 2017

19. Kowalczyk JE, Benoit I, de Vries RP (2014) Chapter two-regulation of plant biomass utilization in Aspergillus. In: Sima S, Geoffrey Michael G (eds) Advances in applied microbiology, vol 88. Academic Press, Cambridge, pp 31-56

20. Kum SJ, Yang SO, Lee SM, Chang PS, Choi YH, Lee JJ, Hurh BS, Kim YS (2015) Effects of Aspergillus species inoculation and their enzymatic activities on the formation of volatile components in fermented soybean paste (doenjang). J Agric Food Chem 63:1401-1418

21. Li H, Handsaker B, Wysoker A, Fennell T, Ruan J, Homer N, Marth G, Abecasis G, Durbin R (2009) The sequence alignment/map format and SAMtools. Bioinformatics 25:2078-2079

22. Liang Y, Pan L, Lin Y (2009) Analysis of extracellular proteins of Aspergillus oryzae grown on soy sauce koji. Biosci Biotechnol Biochem 73:192-195

23. Lioe HN, Selamat J, Yasuda M (2010) Soy sauce and its umami taste: a link from the past to current situation. J Food Sci 75:R71-R76

24. Lombard V, Golaconda Ramulu H, Drula E, Coutinho PM, Henrissat B (2013) The carbohydrate-active enzymes database (CAZy) in 2013. Nucleic Acids Res 42:D490-D495

25. Lu Y, Chen X, Jiang M, Lv X, Rahman N, Dong M, Yan G (2009) Biogenic amines in Chinese soy sauce. Food Control 20:593-597

26. Machida M, Asai K, Sano M, Tanaka T, Kumagai T, Terai G, Kusumoto K, Arima T, Akita O, Kashiwagi Y, Abe K, Gomi K, Horiuchi H, Kitamoto K, Kobayashi T, Takeuchi M, Denning D, Galagan J, Nierman W, Yu J, Archer D, Bennett J, Bhatnagar D, Cleveland T, Fedorova N, Gotoh O, Horikawa H, Hosoyama A, Ichinomiya M, Igarashi R, Iwashita K, Juvvadi P, Kato M, Kato Y, Kin T, Kokubun A, Maeda H, Maeyama N, Maruyama J, Nagasaki H, Nakajima T, Oda K, Okada K, Paulsen I, Sakamoto K, Sawano T, Takahashi M, Takase K, Terabayashi Y, Wortman J, Yamada O, Yamagata Y, Anazawa H, Hata Y, Koide Y, Komori T, Koyama Y, Minetoki T, Suharnan S, Tanaka A, Isono K, Kuhara S, Ogasawara N, Kikuchi H (2005) Genome sequencing and analysis of Aspergillus oryzae. Nature 438:1157-1161

27. MacPherson S, Larochelle M, Turcotte B (2006) A fungal family of transcriptional regulators: the zinc cluster proteins. Microbiol Mol Biol Rev 70:583-604

28. Maeda H, Sano M, Maruyama Y, Tanno T, Akao T, Yotsuka Y, Endo M, Sadurada R, Yamagata Y, Machida M, Akita O, Hasegawa F, Abe K, Gomi K, Nakajima T, Iguchi Y (2004) Transcriptional analysis of genes for energy catabolism and hydrolytic enzymes in the filamentous fungus Aspergillus oryzae using cDNA microarrays and expressed sequence tags. Appl Microbiol Biotechnol 65:74-83

29. Masai K, Maruyama JI, Sakamoto K, Nakajima H, Akita O, Kitamoto K (2006) Square-plate culture method allows detection of differential gene expression and screening of novel, region-specific genes in Aspergillus oryzae. Appl Microbiol Biotechnol 71:881-891

30. Mendes-Pinto MM (2009) Carotenoid breakdown products the-norisoprenoids-in wine aroma. Arch Biochem Biophys 483:236-245

31. Ogawa M, Tokuoka M, Jin FJ, Takahashi T, Koyama Y (2010) Genetic analysis of conidiation regulatory pathways in koji-mold Aspergillus oryzae. Fungal Genet Biol 47:10-18

32. Ogawaa M, Kobayashi T, Koyamaa Y (2012) ManR, a novel $\mathrm{Zn}$ (II)2Cys6 transcriptional activator, controls the $\beta$-mannan utilization system in Aspergillus oryzae. Fungal Genet Biol 49:987-995

33. O'toole DK (1997) The role of microorganisms in soy sauce production. Adv Appl Microbiol 45:87-152

34. Peñalva MA, Tilburn J, Bignell E, Arst HN Jr (2008) Ambient pH gene regulation in fungi: making connetions. Trends Microbiol 16:291-300

35. Rawlings ND, Barrett AJ, Finn R (2016) Twenty years of the MEROPS database of proteolytic enzymes, their substrates and inhibitors. Nucleic Acids Res 44:D343-D350
36. Samson RA, Visagie CM, Houbraken J, Hong SB, Hubka V, Klaassen CH, Perrone G, Seifect KA, Susca A, Tanney JB, Varga J, Kocsubé S, Szigeti G, Yaguchi T, Frisvad JC (2014) Phylogeny, identification and nomenclature of the genus Aspergillus. Stud Mycol 78:141-173

37. Slattery MG, Liko D, Heideman W (2006) The function and properties of the Azfl transcriptional regulator change with growth conditions in Saccharomyces cerevisiae. Eukaryot Cell 5:313-320

38. Steinhaus $P$, Schieberle $P$ (2007) Characterization of the key aroma compounds in soy sauce using approaches of molecular sensory science. J Agric Food Chem 55:6262-6269

39. Tanaka M, Yoshimura M, Ogawa M, Koyama Y, Shintani T, Gomi $\mathrm{K}$ (2016) The $\mathrm{C} 2 \mathrm{H} 2$-type transcription factor, $F l b C$, is involved in the transcriptional regulation of Aspergillus oryzae glucoamylase and protease genes specifically expressed in solid-state culture. Appl Microbiol Biotechnol 100:5859-5868

40. te Biesebeke R, Record E, Van Biezen N, Heerikhuisen M, Franken A, Punt PJ, Van Den Hondel CAMJJ (2005) Branching mutants of Aspergillus oryzae with improved amylase and protease production on solid substrates. Appl Microbiol Biotechnol 69:44-50

41. te Biesebeke R, van Biezen N, De Vos WM, Van Den Hondel CAMJJ, Punt PJ (2005) Different control mechanisms regulate glucoamylase and protease gene transcription in Aspergillus oryzae in solid-state and submerged fermentation. Appl Microbiol Biotechnol 67:75-82

42. Trapnell C, Roberts A, Goff L, Pertea G, Kim D, Kelley DR, Pimentel H, Salzberg SL, Rinn JL, Pachter L (2012) Differential gene and transcript expression analysis of RNA-seq experiments with TopHat and Cufflinks. Nat Protoc 7:562-578

43. Umemura M, Koike H, Yamane N, Koyama Y, Satou Y, Kikuzato I, Teruya M, Tsukahara M, Imada Y, Wachi Y, Miwa Y, Yano S, Tamano K, Kawarabayasi Y, Fujimori K, Machida M, Hirano T (2012) Comparative genome analysis between Aspergillus oryzae strains reveals close relationship between sites of mutation localization and regions of highly divergent genes among Aspergillus species. DNA Res 19:375-382

44. Wang B, Guo G, Wang C, Lin Y, Wang X, Zhao M, Guo Y, He M, Zhang Y, Pan L (2010) Survey of the transcriptome of Aspergillus oryzae via massively parallel mRNA sequencing. Nucleic Acids Res 38:5075-5087

45. Wang G, Keasling DJ (2001) Isolation and characterization of two key regulatory enzyme genes involved in isoprenoid metabolic biosynthesis of Aspergillus nidulans, abstr p 471. Abstr 21st Fungal Genetics Conference. Genetics Society of America, Pacific Grove

46. Woods K, Höfken T (2016) The zinc cluster proteins Upc2 and Ecm 22 promote filamentation in Saccharomyces cerevisiae by sterol biosynthesis-dependent and -independent pathways. Mol Microbiol 99:512-527

47. Wu S, Zhu Z, Fu L, Niu B, Li W (2011) WebMGA: a customizable web server for fast metagenomic sequence analysis. BMC Genom $12: 444$

48. Xu D, Pan L, Zhao H, Zhao M, Sun J, Liu D (2011) Breeding and identification of novel koji molds with high activity of acid protease by genome recombination between Aspergillus oryzae and Aspergillus niger. J Ind Microbiol Biotechnol 38:1255-1265

49. Zhang Y, Tao W (2009) Flavor and taste compounds analysis in Chinese solid fermented soy sauce. Afr J Biotechnol 8:673-681

50. Zhao G, Hou L, Yao Y, Wang C, Cao X (2012) Comparative proteome analysis of Aspergillus oryzae 3.042 and A. oryzae 100-8 strains: towards the production of different soy sauce flavors. J Proteom 75:3914-3924

51. Zhao G, Yao Y, Qi W, Wang C, Hou L, Zeng B, Cao X (2012) Draft genome sequence of Aspergillus oryzae strain 3.042. Eukaryot Cell 11:1178 